\title{
Interfacing Superconducting Qubits and Single Optical Photons Using Molecules in Waveguides
}

\author{
Sumanta Das, ${ }^{1}$ Vincent E. Elfving, ${ }^{1}$ Sanli Faez, ${ }^{2}$ and Anders S. Sørensen ${ }^{1}$ \\ ${ }^{1}$ Niels Bohr Institute, University of Copenhagen, Blegdamsvej 17, 2100 Copenhagen Ø, Denmark \\ ${ }^{2}$ Debye Institute for Nanomaterials Science and Center for Extreme Matter and Emergent Phenomena, \\ Utrecht University, 3584 CC Utrecht, Netherlands \\ (Received 21 July 2016; revised manuscript received 30 January 2017; published 3 April 2017)
}

\begin{abstract}
We propose an efficient light-matter interface at optical frequencies between a single photon and a superconducting qubit. The desired interface is based on a hybrid architecture composed of an organic molecule embedded inside an optical waveguide and electrically coupled to a superconducting qubit placed near the outside surface of the waveguide. We show that high fidelity, photon-mediated, entanglement between distant superconducting qubits can be achieved with incident pulses at the single photon level. Such a low light level is highly desirable for achieving a coherent optical interface with superconducting qubit, since it minimizes decoherence arising from the absorption of light.
\end{abstract}

DOI: 10.1103/PhysRevLett.118.140501

Rapid progress in engineering and control of their physical properties have made superconducting (SC) qubits one of the most promising candidates for future quantum processors [1-4]. If such processors are connected together into a quantum internet [5], it would allow immense applications ranging from secure communication over long distances [6-8] to distributed quantum computation [9-11] and advanced protocols for distributed sensing and atomic clocks [12]. Quantum communication over long distances can, however, only be accomplished through optical means, making it a necessity to build light-matter interfaces at optical frequencies $[5,13]$. This has stimulated immense interest in devising ways of efficiently coupling optical photons to SC systems [14-29]. Tremendous success have been achieved in coupling photons to SC qubits at microwave frequencies [30,31], while in the optical domain only limited indirect coupling has been achieved using transducers [32-34]. Coherent coupling of quantum fields at optical frequencies to a SC system thus remains an outstanding challenge. A principle obstacle to this is the large mismatch between the energy scales of an optical photon $(\sim 1 \mathrm{eV})$ and a SC qubit $(\sim 100 \mu \mathrm{eV})$ [30] making the absorption of even a single optical photon a major disturbance for a SC system. In fact, such effects are used in SC detectors for detection of optical photons [35]. To suppress such disturbances it is therefore highly desirable to keep the number of optical photons to a minimum.

In this Letter we propose a scheme to interface optical photons with a SC qubit at light levels involving only a single or a few photons. To achieve this we introduce a hybrid solid-state architecture depicted in Fig. 1(a) comprising a molecule embedded in an optical waveguide with a SC qubit fabricated near its surface $(\sim 100-500 \mathrm{~nm})$. In comparison to the magnetic coupling considered previously [23-25,31, 36-41], a key feature of our scheme is the electric coupling between the molecule and SC qubit. The coupling strength can then be orders of magnitude stronger, thus allowing for strong coupling in the system. As the SC qubit we consider a Cooper pair box (CPB) where the two quantum states are defined by a single Cooper pair being on each of two superconducting islands. As the Cooper pair oscillates between the islands, it generates a variation in the electric field at the molecule. If the molecule has a large difference in the dipole moment between its ground and excited states, the electric field variation will lead to different Stark shifts of

(a)

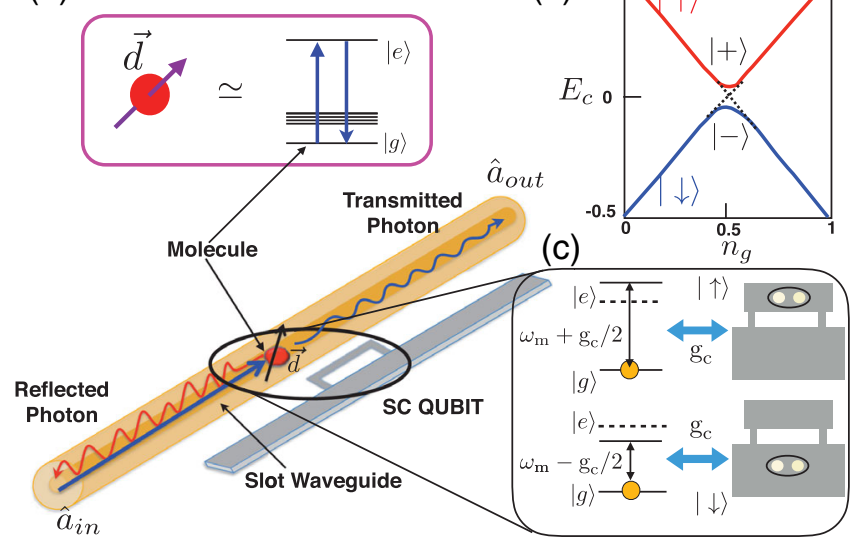

FIG. 1. Schematic of the hybrid molecule-SC system. (a) An organic molecule located inside the optical waveguide is electrically coupled to a SC via a Stark shift. Incident probe photons in the waveguide are elastically scattered by the optical transition of the molecule. Because of the coupling of the SC qubit and the molecule, reflected and transmitted photons are entangled with the internal state of the qubit. (b) The energy levels of a CPB can be represented by two hybridized levels. (c) Oscillation of the Cooper pair between the SC islands leads to shifts of the molecular resonance. 
the energy levels [Fig. 1(c)]. This leads to a sizable shift of the resonance frequency of the molecule, which can be larger than its linewidth, leading to coherent coupling between the molecule and the qubit. Since the molecule is embedded in a waveguide, the shift can lead to measurable effects even for light pulses containing few photons. This is a major advantage over existing hybrid proposals that requires strong optical fields [2,14,26,28,31,36-42], which will lead to decoherence due to quasiparticles created by photon absorption [43].

We show how the achieved light-matter interface allows efficient optical readout of a CPB qubit. Furthermore, we present a detailed scheme for photon-mediated entanglement between two distant SC qubits using hybrids with two molecules at each site. The dipole-dipole interaction between these molecules leads to flip-flop processes between them. This induces an oscillating electric field which can drive a resonant transition in the SC qubit. In total this leads to a Raman process, where the emission of an optical Stokes photon is correlated with the excitation of the SC qubit. Combining the output of two such processes at a beam splitter and conditioning on a click in a detector allows for long distance entanglement between the SC qubits. This opens the possibility of connecting distant SC quantum computers in a large scale quantum network through teleportation.

The key element in the interface is envisioned to be an organic dye molecule. Such molecules can have large differential Stark shift corresponding to a difference in the static dipole moment of one Debye between the ground and excited state $[44,45]$ and can be embedded in optical waveguides [46-49]. For organic molecules all the desired properties have thus been demonstrated experimentally, but the molecule could be replaced by any emitter with similar properties. Placing an ideal two level emitter in an optical waveguide, in principle, allows for coupling efficiencies to optical photons of more than 95\% [50,51]. In practice, a coupling efficiency of $10 \%$ has been measured [47]. For the applications proposed in this Letter, we show that this is sufficient to achieve operations with few photons per pulse $(\lesssim 1)$.

A CPB resembles a two level system [Fig. 1(b)] and can be coherently manipulated at temperatures $\leq 100 \mathrm{mK}$ [52-54]. The Hamiltonian of this system can be written as $\mathcal{H}_{\mathrm{cp}}=-\frac{1}{2}\left(\chi_{1} \eta_{z}+\chi_{2} \eta_{x}\right)$, where $\chi_{1,2}$ can be externally controlled while $\eta_{z}, \eta_{x}$ are the Pauli spin-1/2 operators defined in the spin basis $\{|\uparrow\rangle,|\downarrow\rangle\}$ corresponding to distinct charge states [52]. The interaction Hamiltonian $\mathcal{H}_{\mathrm{I}}$ of the molecule-qubit hybrid governing the coherent dynamics can be written in the form [55]

$$
\mathcal{H}_{\mathrm{I}}=\frac{\hbar g_{m}}{2} \sigma^{+} \hat{a} e^{-i \omega_{p} t}+\text { H.c. }+\frac{1}{4} \hbar g_{c} \eta_{z} \otimes\left(\sigma^{z}+\llbracket\right) .
$$

Here the first term and its Hermitian conjugate correspond to the light-molecule interaction, while the last term is the
molecule-CPB interaction. The operators $\sigma^{z}, \sigma^{ \pm}$are the standard dipole transition operators for a two level system and $\hat{a}$ is the field operator of the incoming photon pulse [59] of central frequency $\omega_{p}$. The incoming light couples to the molecule with a strength $g_{m}=\vec{\wp}_{e g} \cdot \overrightarrow{\mathcal{F}} / \hbar$, where $\vec{\wp}_{e g}$ is the dipole moment of the optical transition $|e\rangle \leftrightarrow|g\rangle$ in the molecule and $\overrightarrow{\mathcal{F}}$ the mode function of the incoming photon in the one-dimensional waveguide. Furthermore, $g_{c}=$ $\Delta \vec{\wp}_{c} \cdot \Delta \overrightarrow{\mathcal{E}} / \hbar$ is the molecule-CPB coupling strength, where $\Delta \vec{\wp}_{c}$ is the difference in the static dipole moments between the excited and ground manifold of the molecule, while $\Delta \overrightarrow{\mathcal{E}}$ is the electrostatic field variation as seen by the molecule due to the tunneling of a single Cooper pair.

We first outline a recipe for detecting the qubit's state by optical photons in a scheme reminiscent of qubit readout in the dispersive regime of circuit QED [60]. We assume that the CPB is operated at a gate voltage away from the charge degeneracy point $\chi_{1} \gg \chi_{2}$, i.e., in the linear regime of Fig. 1(b). Working in this regime, the eigenstates of the qubit Hamiltonian $\mathcal{H}_{\mathrm{cp}}$ are the $\eta^{z}$ eigenstates which are (first order) sensitive to the interaction. The CPB-molecule interaction Hamiltonian (1) reveals that the state of the qubit shifts the excited state of the molecule by $\pm \frac{1}{2} \hbar g_{c}$, compared to the unperturbed resonance at $\omega_{m}$ [Fig. 1(c)], making it sensitive to the qubit state. The molecular resonance line is thus split into two, corresponding to the two qubit states $\{|\uparrow\rangle,|\downarrow\rangle\}$, with the splitting given by the molecule-qubit coupling $g_{c}$. We can, therefore, determine the qubit state by studying the scattering of an incoming photons and measuring whether they are transmitted or reflected.

Considering a small CPB [54], the coupling can be estimated from the field of a point charge sitting at the edge of a semi-infinite medium. This gives $|\Delta \mathcal{E}| \simeq(7-19) \mathrm{kV} / \mathrm{m}$, at the location of the molecule in a polyethylene waveguide of permittivity $\sim 2.3$ [61], due to the presence of a Cooper pair on an island situated about $\sim(500-300) \mathrm{nm}$ away [62]. However, in reality, due to the size of the qubit and the composition of the waveguide, the electric field is smaller. We find from explicit numerical simulation a value of $|\Delta \overrightarrow{\mathcal{E}}| \simeq$ $(4.5-16) \mathrm{kV} / \mathrm{m}$ for an island situated $\sim(500-125) \mathrm{nm}$ away from the surface of the waveguide [55]. Hence, for $\left|\Delta \vec{\wp}_{c}\right|=1 \mathrm{D}[44,45]$, we can obtain a coupling strength of $g_{c} \sim(2 \pi) \times(25-80) \mathrm{MHz}$. As organic molecules typically have optical transitions with narrow linewidths $\gamma \sim$ $(2 \pi) \times 20 \mathrm{MHz}[44,46]$, we can achieve a strong coupling regime $\left(g_{c}>\gamma\right)$ where the molecular line splitting exceeds the linewidth. Thus the two internal states of the qubit can be distinguished by sending in a pulse resonant with one of the resonance peaks and measuring whether photons are reflected. At resonance, we evaluate the reflection probability [55] to be $\left(\gamma_{1 \mathrm{D}} / \gamma\right)^{2}$, where $\gamma_{1 \mathrm{D}}$ is the decay rate into the 1D waveguide. Hence, we can distinguish the two states 



FIG. 2. (a) Schematics of Raman configuration. The molecular levels $\left|e_{1} g_{2}\right\rangle$ and $\left|g_{1} e_{2}\right\rangle$ are hybridized by the dipole-dipole interaction to form the dressed states $|A\rangle$ and $|S\rangle$, the separation of which is tuned into resonance with the qubit frequency $\omega_{q}$. A photon scattered along the transition $|g,-\rangle \rightarrow|S,-\rangle$ is emitted as a Stokes photon along the transition $|A,+\rangle \rightarrow|g,+\rangle$ due to resonant coupling among the states $|S,-\rangle \leftrightarrow|A,+\rangle$. (b) Normalized probability of Raman scattering derived in Eq. (3) for a single incident photon as a function of coupling $\left(g_{c_{1}}-g_{c_{2}}\right) / \gamma$ for different values of the dipolar coupling strength. Here we have assumed $\gamma_{c} / \gamma_{i}=1$.

by sending in $\left(\gamma / \gamma_{1 \mathrm{D}}\right)^{2} \sim 100$ photons for the experimentally observed efficiency of $\gamma_{1 \mathrm{D}} / \gamma=0.1$ [47].

To achieve a coherent interface we chose the qubit to be at the so-called sweet spot $\left(\chi_{1}=0\right)$, where the energy of the eigenstates $| \pm\rangle=(|\uparrow\rangle \pm|\downarrow\rangle) / \sqrt{2}$, of the CPB Hamiltonian are first order insensitive to charge noise [Fig. 1(b)] and thus can have long coherence time $[63,64]$. To be able to work with a few photons we consider the Raman scattering scheme shown in Fig. 2(a). This is realized by having two organic molecules with properties as above and with optical transitions of nearly the same frequencies, e.g., by tuning them into resonance using an external field. The molecules are assumed to have a separation less than the optical wavelength, and thus couple to each other via near field optical dipolar interaction [65]. Furthermore, we assume a reflector at one end of the waveguide such that the waveguide is single sided to maximize the collection of Raman photons.

The interaction Hamiltonian $\mathcal{H}_{I}$ comprises the dipolar coupling Hamiltonian $\mathcal{H}_{\mathrm{dd}}=\hbar V\left(\sigma_{1}^{+} \sigma_{2}^{-}+\sigma_{2}^{+} \sigma_{1}^{-}\right)$of strength $V$, and the Hamiltonian

$$
\mathcal{H}_{c}=\sum_{j}\left(\frac{\hbar g_{m_{j}}}{2} \sigma_{j}^{+} \hat{a} e^{-i \omega_{p} t}+\text { H.c. }+\frac{\hbar g_{c_{j}}}{4} \eta_{z} \otimes\left(\sigma_{j}^{z}+\mathbb{\square}\right)\right),
$$

where $g_{m_{j}}$ and $g_{c_{j}}$ for $(j=1,2)$ corresponds to the coupling strength of the incoming light and CPB to the molecules, respectively. The strong dipole-dipole interaction $\mathcal{H}_{\mathrm{dd}}$ can be diagonalized to form two dressed state $|S\rangle$ and $|A\rangle$, which are split by $2 \mathcal{V}=\sqrt{4 V^{2}+\delta_{0}^{2}}$ and have an electrical dipole transition between them. Using an external field to vary the difference in the molecular energies $\delta_{0}=\left(\omega_{m_{1}}-\omega_{m_{2}}\right)$, the transition between the dressed states can be brought into resonance with the qubit transition, $2 \mathcal{V}=\omega_{q}$. This resonance condition allows the exchange of energy between the qubit and the excited manifold of the molecules through the interaction of the dipoles with the charge fluctuations in the CPB. This enables the Raman transition $|g,-\rangle \rightarrow$ $|S,-\rangle \rightarrow|A,+\rangle \rightarrow|g,+\rangle$ [Fig. 2(a)]. Here the molecular system starts and ends in the joint ground state $|g\rangle=\left|g_{1}, g_{2}\right\rangle$, while the qubit is flipped from state $|-\rangle$ to $|+\rangle$ by the emission of a Stokes photon of frequency $\omega_{s}=\left(\omega_{p}-\omega_{q}\right)$. The effective coupling constant between the states $|S,-\rangle$ and $|A,+\rangle$, which enables this transition, is given by $\mathcal{G}=\left(g_{c_{1}}-g_{c_{2}}\right) V / \sqrt{4 V^{2}+\delta_{0}^{2}}$. Using the effective operator formalism [66], we find that at resonance the probability for an incident single photon to induce a Raman scattering into the waveguide for moderate coupling $g_{c_{1,2}}^{2} / \gamma \omega_{q}<1$ is $\mathcal{P}_{R}=\left(\gamma_{1 \mathrm{D}} / \gamma\right)^{2} \wp_{R}$, where [55],

$$
\wp_{R}=\left(\frac{\delta_{0}}{\omega_{q}}\right)^{2}\left(\frac{4 \mathcal{G}^{2}}{\Gamma_{s}^{2} \Gamma_{a}^{2} / 4 \gamma^{2}+4 \mathcal{G}^{2}}\right) .
$$

Here, $\gamma=\gamma_{1 \mathrm{D}}+\gamma_{c}+\gamma_{i}$ is the total decay rate of each molecule (assumed identical for the two molecules), $\Gamma_{s}=$ $\gamma+2 \gamma_{c} V / \omega_{q}, \Gamma_{a}=\gamma-2 \gamma_{c} V / \omega_{q}$ are the decay rates of $|S\rangle$ and $|A\rangle$, respectively, $\gamma_{i}$ is the intrinsic decay rate of each molecule while $\gamma_{c}$ is the collective decay rate of the molecules. In deriving the Raman scattering probability we assumed that the molecules have the same $\gamma_{1 \mathrm{D}}$ and that they are close enough that we can ignore phases in the collective decay [67] arising from the spatial positions of the molecules. The probability of Raman scattering is much larger than the reverse process $|A,+\rangle \rightarrow|S,-\rangle$, which is suppressed by a factor $(\mathcal{G} \gamma)^{2} / \omega_{q}^{4}$ since it is off resonant [55].

In Fig. 2(b) we plot the Raman probability as a function of $\left(g_{c_{1}}-g_{c_{2}}\right) / \gamma$ for different ratios of $V / \omega_{q}$. The results can be understood from the need to have both good hybridization and coupling to the waveguide. For low $V / \omega_{q}$ the hybridization of $\left|e_{1} g_{2}\right\rangle$ and $\left|g_{2} e_{1}\right\rangle$ to $|A\rangle$ and $|S\rangle$ is small, which limits the coupling, whereas for $V / \omega_{q} \rightarrow 1 / 2$, $\delta_{0} \rightarrow 0,|A\rangle$ becomes a dark state of the coupling to the waveguide so that $\mathcal{P}_{R} \rightarrow 0$. For $V / \omega_{q} \gtrsim 0.1$, the probability quickly reaches its maximum value even for limited coupling strengths $\left(g_{c_{1}}-g_{c_{2}}\right) / \gamma \gtrsim 1$, whereas saturation is slower for weaker dipole coupling due to the lack of hybridization. We find from Fig. 2(b), that for a feasible $V / \omega_{q}=0.2$ and a moderate coupling strength $\left(g_{c_{1}}-g_{c_{2}}\right) / \gamma=4$, the Raman scattering probability is $\mathcal{P}_{R} \simeq$ $0.77 \times\left(\gamma_{1 \mathrm{D}} / \gamma\right)^{2}$ (note that since the $g_{c_{1}}$ and $g_{c_{2}}$ can have opposite signs, their difference can exceed their individual values). These parameters will be used for all numerical examples below. The value of $\mathcal{P}_{R}$ is close to its upper limit of $\left(\gamma_{1 \mathrm{D}} / \gamma\right)^{2}$ set by the necessity of having both waveguide absorption and emission by the molecule. Furthermore, 


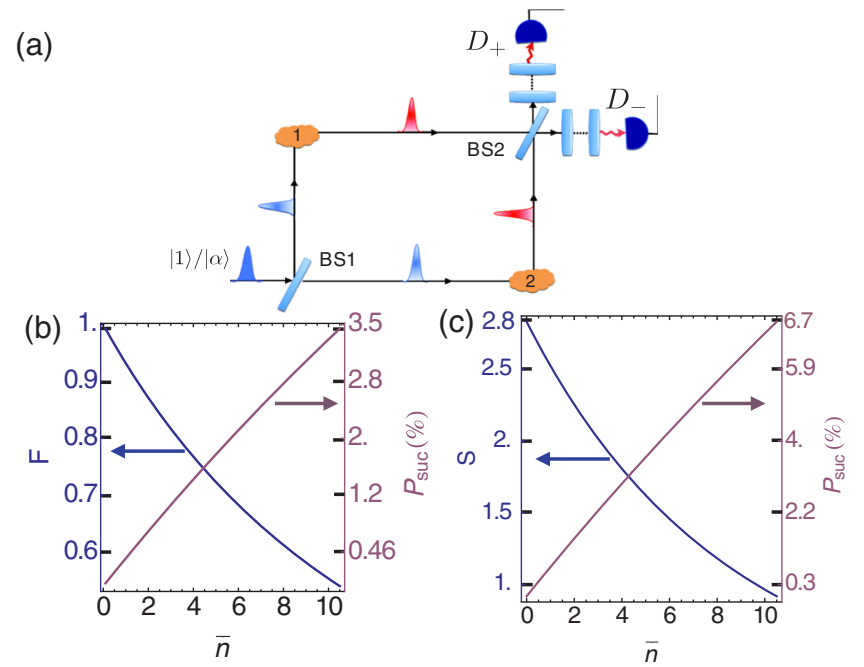

FIG. 3. (a) Interferometric scheme to generate the maximally entangled Bell state $\left|\Psi_{+}\right\rangle$between two SC qubits using a single photon $|1\rangle$ or coherent state $|\alpha\rangle$ as input. Generation of entanglement is conditioned on a click of detector $D_{ \pm}$. (b) Fidelity $F$ and success probability $P_{\text {suc }}^{(c)}$ for Bell state generation with a pulse with $\bar{n}$ photons on average. (c) Bell parameter $S$ and success probability $P_{\text {suc }}^{(c)}$ for an entangled state between a single qubit and a photon, for an incident pulse of $\bar{n}$ photons. For all the plots we have assumed $\gamma_{1 \mathrm{D}} / \gamma=0.1, \eta=50 \%,\left(g_{c_{1}}-g_{c_{2}}\right) / \gamma=4$, $\mathcal{P}_{R}=0.77 \times\left(\gamma_{1 \mathrm{D}} / \gamma\right)^{2}$, and $\gamma_{c} / \gamma=\gamma_{i} / \gamma=0.45$.

the Raman probability is not very sensitive to the precise value of the dipole coupling, making it attractive even for randomly placed molecules.

The effective Raman scheme allows using the interferometric framework [68,69], shown schematically in Fig. 3(a) to generate entanglement between distant (e.g., 10's kms) SC qubits via the detection of a photon. For this purpose we assume that both hybrids are initialized in state $|g,-\rangle_{1(2)}$. An incident photon pulse [blue in Fig. 3(a)] is split by the beam splitter and sent towards the two interfaces, where it can induce Raman transitions. An outgoing photon (red) is correlated with a transition to $|+\rangle$ in the corresponding qubit. Interfering the outputs on a beam splitter erases the "which way" information about which the interface emitted the photon. Hence, by conditioning on clicks in detectors $D_{ \pm}$after frequency filtering out photons, which have not undergone Raman scattering, the qubits are projected into one of the maximally entangled Bell states $\left|\Psi_{ \pm}\right\rangle=$ $(1 / \sqrt{2})|g\rangle\left(|-\rangle_{1}|+\rangle_{2} \pm|+\rangle_{1}|-\rangle_{2}\right)$ depending on which detector clicks. With single incident photons this process creates ideal Bell states, provided that no other sources of noise are present.

For simplicity, we consider the two hybrids to have equivalent physical properties and work in the limit of moderate coupling $g_{c_{1,2}}^{2} / \gamma \omega_{q}<1$. Assuming the input pulse to be a single photon, we find that within the present model the process has a fidelity $F=1$, and a success probability of $P_{\text {suc }}^{(1)}=\eta \mathcal{P}_{R}$, where $\eta$ is the photodetection efficiency of the single photon detectors [55] [in reality $F<1$, due to, e.g., dephasing of the qubits during the time needed for scattering of photons (see below)]. For the set of parameters used above along with $\gamma_{1 \mathrm{D}} / \gamma=0.1$, we get $P_{\text {suc }}^{(1)} \simeq 3.8 \times 10^{-3}$, for $\eta=50 \%$.

A simpler experimental method is to use a weak coherent state as input. Assuming identical hybrids and an intensity below saturation, we find the conditional fidelity for an incident photon pulse with $\bar{n}$ photons in the lowest order to be $F=1-(\bar{n} / 2)\left(\mathcal{P}_{R}+\mathcal{P}_{R O}+\mathcal{P}_{D} / 4\right)$, with the corresponding success probability [55]

$$
P_{\text {suc }}^{(c)}=2 P_{\text {suc }}^{(1)}\left\{\frac{1-e^{-\frac{\pi}{2}\left(\mathcal{P}_{R}+\mathcal{P}_{R O}\right)}}{\mathcal{P}_{R}+\mathcal{P}_{R O}}\right\},
$$

where the probability of Raman scattering to the outside (not into the waveguide) is $\mathcal{P}_{R O}=\left(\gamma_{1 \mathrm{D}} / \gamma\right)\left\{\left(\gamma_{c} / \gamma\right) \times\right.$ $\left.\left(\delta_{0} / \omega_{q}\right)^{2}+\left(\gamma_{i} / \gamma\right)\left[1+\left(2 V / \omega_{q}\right)\right]\right\}\left[\left(2 \mathcal{G}^{2}\right) /\left(\Gamma_{s}^{2} \Gamma_{a}^{2} / 4 \gamma^{2}+4 \mathcal{G}^{2}\right)\right]$, while $\mathcal{P}_{D}$ is the probability of dephasing induced by elastic scattering [55]. As an example, for $\bar{n}=1.5$ we get a success probability $P_{\text {suc }}^{(c)} \simeq 5.6 \times 10^{-3}$ for creating a Bell state with fidelity $F \sim 90 \%$. Thus, for an input coherent state, we gain substantially in experimental simplicity with limited reduction in fidelity. We show in Fig. 2(b) the behavior of $F$ and $P_{\text {suc }}^{(c)}$ as a function of the mean photon numbers.

A first step towards the above entanglement generation scheme can be achieved in a simpler setup involving only a single SC qubit, by replacing the other hybrid in the interferometer in Fig. 3(a) with a frequency shifter. In this case, one can obtain a violation of the CHSH inequality $S \leq 2$ [70,71], where $S$ is the violation parameter between the qubit and a single photon detected at $D_{ \pm}$[55]. In Fig. 3(c) we show the behavior of $S$ and $P_{\text {suc }}^{(c)}$ as a function of the mean photon number. Using the above parameters we find $S \geq 2.3$ for $\bar{n}=2$, with a corresponding success probability $P_{\text {suc }}^{(c)} \simeq$ $1.5 \%$ for $\eta=50 \%$.

So far we have ignored the time $T$ it takes to perform the light scattering. The scattering needs to be completed within the coherence time of the qubit. Since we condition on photon detection, the interaction strength only enters through the success probability as contained in Eq. (3). The time is therefore only limited by the requirement to be resonant with states of width $\gamma$, implying $\gamma T \gg 1$. With $\gamma=(2 \pi) 20 \mathrm{MHz}$ we can chose a pulse duration $T=50 \mathrm{~ns}$. Since CPBs of the type considered here have shown coherence times of $T_{2}=500 \mathrm{~ns}$ [72] with a Gaussian decay, we estimate a further reduction of the fidelity by $\left(T / T_{2}\right)^{2}<1 \%$ due to qubit decoherence [55].

An attractive feature of the Raman scheme is that it relies on an electric coupling at the qubit resonance frequency. This scheme can thus be extended to more advanced qubit 
designs, such as transmons, which are insensitive to low frequency electric noise and thus have very long coherence times [64]. The larger size of these qubits, however, diminishes the coupling, making it infeasible for the molecular parameters considered here. This could possibly be overcome with qubit designs optimized for this purpose or by using other emitters with larger dipole moments. Alternatively one can envision dedicated CPB qubits acting as communication interfaces for quantum computers based on transmons.

In conclusion, we have proposed a novel hybrid system formed by an organic molecule embedded in an optical waveguide and electrically coupled to a SC qubit, that provides a light-matter interface for quantum information transfer over long distances. This could open new directions in quantum communication using SC quantum processors in a network.

S. D., V.E., and A. S. gratefully acknowledge financial support from the European Union Seventh Framework Programme ERC Grant QIOS (Grant No. 306576) and the Danish council for independent research (Natural Sciences). S. F. acknowledges support by the European Research Council, Project No. 279248.

[1] R. J. Schoelkopf and S. M. Girvin, Nature (London) 451, 664 (2008).

[2] M. H. Devoret and R. J. Schoelkopf, Science 339, 1169 (2013).

[3] J. Kelly et al., Nature (London) 519, 66 (2015).

[4] A. D. Crcoles, E. Magesan, S. J. Srinivasan, A. W. Cross, M. Steffen, J. M. Gambetta, and J. M. Chow, Nat. Commun. 6, 6979 (2015).

[5] H. J. Kimble, Nature (London) 453, 1023 (2008).

[6] H.-J. Briegel, W. Dür, J. I. Cirac, and P. Zoller, Phys. Rev. Lett. 81, 5932 (1998).

[7] N. Sangouard, C. Simon, H. de Riedmatten, and N. Gisin, Rev. Mod. Phys. 83, 33 (2011).

[8] S. Muralidharan, L. Li, J. Kim, N. Lütkenhaus, M. D. Lukin, and L. Jiang, Sci. Rep. 6, 20463 (2016).

[9] J. I. Cirac, A. K. Ekert, S. F. Huelga, and C. Macchiavello, Phys. Rev. A 59, 4249 (1999).

[10] C. Crepeau, D. Gottesman, and A. Smith, arXiv:quant-ph/ 0206138

[11] M. Ben-Or, C. Crepeau, D. Gottesman, A. Hassidim, and A. Smith, Proceedings of the 47th Annual IEEE Symposium on Foundations of Computer Science (FOCS'06) 0-7695-2720 (IEEE Computer Society, Washington, D.C., 2006).

[12] P. Kómár, E. M. Kessler, M. Bishof, L. Jiang, A. S. S rensen, J. Ye, and M. D. Lukin, Nat. Phys. 10, 582 (2014).

[13] N. Gisin, G. Ribordy, W. Tittel, and H. Zbinden, Rev. Mod. Phys. 74, 145 (2002).

[14] A. S. Sørensen, C. H. van der Wal, L. I. Childress, and M. D. Lukin, Phys. Rev. Lett. 92, 063601 (2004).

[15] P. Rabl, D. DeMille, J. M. Doyle, M. D. Lukin, R. J. Schoelkopf, and P. Zoller, Phys. Rev. Lett. 97, 033003 (2006).
[16] A. Andre, D. DeMille, J. M. Doyle, M. D. Lukin, S. E. Maxwell, P. Rabl, R. J. Schoelkopf, and P. Zoller, Nat. Phys. 2, 636 (2006).

[17] M. Wallquist, K. Hammerer, P. Rabl, M. Lukin, and P. Zoller, Phys. Scr. T137, 014001 (2009).

[18] J. M. Taylor, A. S. Sørensen, C. M. Marcus, and E. S. Polzik, Phys. Rev. Lett. 107, 273601 (2011).

[19] S. Barzanjeh, M. Abdi, G. J. Milburn, P. Tombesi, and D. Vitali, Phys. Rev. Lett. 109, 130503 (2012).

[20] L. Tian, Phys. Rev. Lett. 108, 153604 (2012).

[21] Y.-D. Wang and A. A. Clerk, Phys. Rev. Lett. 108, 153603 (2012).

[22] Z-L. Xiang, S. Ashhab, J. Q. You, and F. Nori, Rev. Mod. Phys. 85, 623 (2013).

[23] D. Marcos, M. Wubs, J. M. Taylor, R. Aguado, M. D. Lukin, and A. S. Sørensen, Phys. Rev. Lett. 105, 210501 (2010).

[24] K. Xia, M. R. Vanner, and J. Twamley, Sci. Rep. 4, 5571 (2014).

[25] C. OBrien, N. Lauk, S. Blum, G. Morigi, and M. Fleischhauer, Phys. Rev. Lett. 113, 063603 (2014).

[26] J. D. Pritchard, J. A. Isaacs, M. A. Beck, R. McDermott, and M. Saffman, Phys. Rev. A 89, 010301 (2014).

[27] L. A. Williamson, Y.-H. Chen, and J. J. Longdell, Phys. Rev. Lett. 113, 203601 (2014).

[28] Z. Q. Yin, W. L. Yang, L. Sun, and L. M. Duan, Phys. Rev. A 91, 012333 (2015).

[29] O. Cernotik and Kl. Hammerer, Phys. Rev. A 94, 012340 (2016).

[30] A. Wallraff, D. I. Schuster, A. Blais, L. Frunzio, R.- S. Huang, J. Majer, S. Kumar, S. M. Girvin, and R. J. Schoelkopf, Nature (London) 431, 162 (2004).

[31] Y. Kubo, F. R. Ong, P. Bertet, D. Vion, V. Jacques, D. Zheng, A. Dréau, J.-F. Roch, A. Auffeves, F. Jelezko, J. Wrachtrup, M. F. Barthe, P. Bergonzo, and D. Esteve, Phys. Rev. Lett. 105, 140502 (2010).

[32] J. Bochmann, A. Vainsencher, D. D. Awschalom, and A. N. Cleland, Nat. Phys. 9, 712 (2013).

[33] R. W. Andrews, R. W. Peterson, T. P. Purdy, K. Cicak, R. W. Simmonds, C. A. Regal, and K. W. Lehnert, Nat. Phys. 10, 321 (2014).

[34] T. Bagci, A. Simonsen, S. Schmid, L. G. Villanueva, E. Zeuthen, J. Appel, J. Taylor, A. S. Sørensen, K. Usami, A. Schliesser, and E. S. Polzik, Nature (London) 507, 81 (2014).

[35] C. M. Natarajan, M. G. Tanner, and R. H. Hadfield, Supercond. Sci. Technol. 25, 063001 (2012).

[36] S. Probst, H. Rotzinger, S. Wünsch, P. Jung, M. Jerger, M. Siegel, A. V. Ustinov, and P. A. Bushev, Phys. Rev. Lett. 110, 157001 (2013).

[37] D. I. Schuster, A. P. Sears, E. Ginossar, L. DiCarlo, L. Frunzio, J. J. L. Morton, H. Wu, G. A. D. Briggs, B. B. Buckley, D. D. Awschalom, and R. J. Schoelkopf, Phys. Rev. Lett. 105, 140501 (2010).

[38] K. Tordrup and K. Mølmer, Phys. Rev. A 77, 020301 (2008).

[39] A. Imamoǵlu, Phys. Rev. Lett. 102, 083602 (2009).

[40] J. Verdú, H. Zoubi, Ch. Koller, J. Majer, H. Ritsch, and J. Schmiedmayer, Phys. Rev. Lett. 103, 043603 (2009).

[41] X. Zhu, S. Saito, A. Kemp, K. Kakuyanagi, S. Karimoto, H. Nakano, W. Munro, Y. Tokura, M. S. Everitt, K. Nemoto, 
M,Kasu, N. Mizuochi, and K. Semba, Nature (London) 478, 221 (2011).

[42] S. Blum, C. O’Brien, N. Lauk, P. Bushev, M. Fleischhauer, and G. Morigi, Phys. Rev. A 91, 033834 (2015).

[43] G. Catelani, R. J. Schoelkopf, M. H. Devoret, and L. I. Glazman, Phys. Rev. B 84, 064517 (2011).

[44] M. Orrit, J. Bernard, A. Zumbusch, and R. I. Personov, Chem. Phys. Lett. 196, 595 (1992).

[45] Ch. Brunel, Ph. Tamarat, B. Lounis, J. C. Woehl, and Michel Orrit, J. Phys. Chem. A 103, 2429 (1999).

[46] Y. L. A. Rezus, S. G. Walt, R. Lettow, A. Renn, G. Zumofen, S. Götzinger, and V. Sandoghdar, Phys. Rev. Lett. 108, 093601 (2012).

[47] S. Faez, P. Türschmann, H. R. Haakh, S. Götzinger, and V. Sandoghdar, Phys. Rev. Lett. 113, 213601 (2014).

[48] M. Gaio, M. Moffa, M. Castro-Lopez, D. Pisignano, A. Camposeo, and R. Sapienza, ACS Nano 10, 6125 (2016).

[49] S. M. Skoff, D. Papencordt, H. Schauffert, and Arno Rauschenbeutel, arXiv:1604.04259.

[50] V. R. Almeida, Qianfan Xu, C. A. Barrios, and M. Lipson, Opt. Lett. 29, 1209 (2004).

[51] Q. Quan, I. Bulu, and M. Lonćar, Phys. Rev. A 80, 011810 (2009).

[52] Y. Makhlin, G. Schoen, and A. Shnirman, Rev. Mod. Phys. 73, 357 (2001).

[53] V. Bouchiat, D. Vion, P. Joyez, D. Esteve, and M. H. Devoret, Phys. Scr. T76, 165 (1998).

[54] D. Vion, A. Aassime, A. Cottet, P. Joyez, H. Pothier, C. Urbina, D. Esteve, and M. H. Devoret, Science 296, 886 (2002).

[55] See Supplemental Material at http://link.aps.org/ supplemental/10.1103/PhysRevLett.118.140501, which includes Refs. [56-58], for details of the qubit state detection, numerical estimate of the molecule-qubit coupling and the conditional fidelity of the generated entangled state.
[56] S. Das, V. Elfving, F. Reiter, and A. S. Sørensen (to be published).

[57] D. Witthaut and A. S. Sørensen, New J. Phys. 12, 043052 (2010).

[58] Y. Li, L. Aolita, D. E. Chang, and L. C. Kwek, Phys. Rev. Lett. 109, 160504 (2012).

[59] M. O. Scully and M.H. Zubairy, Quantum Optics (Cambridge University Press, Cambridge, England, 1997).

[60] A. Blais, R.-S. Huang, A. Wallraff, S. M. Girvin, and R. J. Schoelkopf, Phys. Rev. A 69, 062320 (2004).

[61] A. C. Wirtz, C. Hofmann, and E. J. J. Groenen, J. Phys. Chem. B 110, 21623 (2006).

[62] S. Faez, S. J. van der Molen, and M. Orrit, Phys. Rev. B 90, 205405 (2014).

[63] G. Ithier, E. Collin, P. Joyez, P. J. Meeson, D. Vion, D. Esteve, F. Chiarello, A. Shnirman, Y. Makhlin, J. Schriefl, and G. Schön, Phys. Rev. B 72, 134519 (2005).

[64] A. A. Houck, J. Koch, M. H. Devoret, S. M. Girvin, and R. J. Schoelkopf, Quantum Inf. Process. 8, 105 (2009).

[65] C. Hettich, C. Schmitt, J. Zitzmann, S. Khn, I. Gerhardt, and V. Sandoghdar, Science 298, 385 (2002).

[66] F. Reiter and A. S. Sørensen, Phys. Rev. A 85, 032111 (2012).

[67] S. Das, G. S. Agarwal, and M. O. Scully, Phys. Rev. Lett. 101, 153601 (2008).

[68] C. Cabrillo, J. I. Cirac, P. Garcia-Fernández, and P. Zoller, Phys. Rev. A 59, 1025 (1999).

[69] L. I. Childress, J. M. Taylor, A. S. Sørensen, and M. D. Lukin, Phys. Rev. A 72, 052330 (2005).

[70] J. F. Clauser, M. A. Horne, A. Shimony, and R. A. Holt, Phys. Rev. Lett. 23, 880 (1969).

[71] A. Aspect, P. Grangier, and G. Roger, Phys. Rev. Lett. 47, 460 (1981).

[72] A. Wallraff, D. I. Schuster, A. Blais, L. Frunzio, J. Majer, M. H. Devoret, S. M. Girvin, and R. J. Schoelkopf, Phys. Rev. Lett. 95, 060501 (2005). 